\title{
Fabrication of acoustic ejectors with replaceable acoustic lens by using soft-lithography
}

\author{
You-Lin Tu, Jin-An Wu, Shih-Jui Chen* \\ Department of Mechanical Engineering \\ National Central University \\ Taoyuan, Taiwan \\ *raychen@cc.ncu.edu.tw
}

\begin{abstract}
This paper presents a novel design of an acousticwave liquid ejector by using PDMS soft-lithography. Both annular patterned lens membrane and piezoelectric transducer are designed to be replaceable to avoid ink clogging on the ejector surface. With the integration of an external siphoning system, the ejector is capable of jetting downward rather than only upward and refilling ink automatically without external force, which makes it suitable for 3D printing applications. By applying the electric signal at $2.4 \mathrm{MHz}$, the ejector can eject water downward with a speed of $0.29 \mathrm{~m} / \mathrm{s}$ and droplet dimension of $720 \mu \mathrm{m}$.
\end{abstract}

Keywords-Acoustic ejector, Soft-lithography

\section{INTRODUCTION}

Many studies on liquid ejectors have been made over the past few decades [1-6]. The thermal bubble type ejects the droplet because of the heat expansion of the air bubble generated by the resistor [1]. The thermal bubble ejector is low-cost but has poor quality and limited life-time due to the cavitation damage[2]. For the thermal diaphragm vibration ejector [3], the diaphragm simultaneously deflects to eject the liquid due to the thermally induced compressive stress generated by the resistor. Electrostatic actuation ejector comprises a silicon pressure plate and a corresponding parallel transparent electrode [4]. By applying a DC voltage, the device could eject and refill the ink through the deflection of pressure plate. Piezoelectric actuation ejector induces abruptly compression in the liquid chamber, causing the ejection of the droplet at a nozzle [5]. However, piezoelectric ejector requires too much power and space to drive the array of nozzles.

Typical acoustic wave liquid ejectors [6] use Fresnel lens to focus the wave produced by the piezoelectric transducer to eject the ink. Without the nozzle, the acoustic wave ejector does not have the problem of clogging. However, the acoustic wave ejector still has shortcomings like the control of the droplet dimension and the height of ink liquid level.

With the popularity and development of $3 \mathrm{D}$ printing and household printers, the demand of solutions that require speed, reliability, and cost efficiency is dramatically increasing recently. The advanced medical therapies such like 3D biomedical printing of tissue or organs [7] also accelerate the innovation. With the high viscosity of the photosensitive resins used in 3D printing, ejecting the materials upward needs to overcome both the surface tension and the gravity [8]. In this paper, a novel

This research was supported by the research grants (MOST 1042221-E-008-018-MY2, MOST 105-2218-E-008-008) of the Ministry of Science and Technology, Taiwan.

\author{
Barthélemy Cagneau, Luc Chassagne \\ Université de Versailles Saint-Quentin en Yvelines \\ UVSQ / LISV \\ Vélizy, France
}

design integrated with acoustic wave liquid ejectors and siphoning structure system is proposed. To avoid the solidified resins damaging the focusing effect, the annular Fresnel lens membrane is replaceable while keeping the same piezoelectric ceramic. The overall ejection system is shown in Fig. 1.

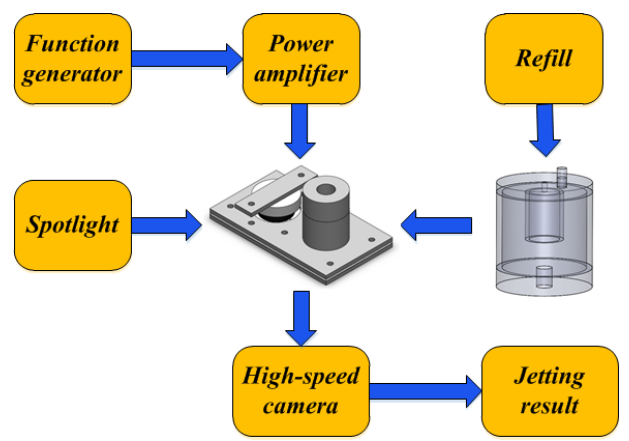

Fig.1 The schematic of the overall ejection system.

\section{THOERY}

The transducer consists of a piezoelectric Lead Zirconate Titanate (PZT) ceramic sandwiched by two nickel electrodes with acoustic lens on top of the electrode [6]. The acoustic lens is patterned into Fresnel half-wave bands (FHWB), and the radii of FHWB sources can be expressed by [9]

$$
r_{n}=\sqrt{2 n \lambda \times\left(F+\frac{n \lambda}{2}\right)},
$$

where $n=1,2,3, \lambda$ is the wavelength of the acoustic wave, and $F$ is the focal length.

The odd and even numbered zones are made of polydimethylsiloxane (PDMS) and air, respectively. Due to acoustic impedance mismatch, the acoustic wave produced by the transducer will pass through the PDMS region and reflect at the air region. The reflectance can be calculated as

$$
R=\left[\frac{\frac{Z_{2}}{\cos \alpha_{2}}-\frac{Z_{1}}{\cos \alpha_{1}}}{\frac{Z_{2}}{\cos \alpha_{2}}+\frac{Z_{1}}{\cos \alpha_{1}}}\right]^{2},
$$

where $Z_{1}$ and $Z_{2}$ are the material's acoustic impedance; $\alpha_{1}$ and $\alpha_{2}$ are the incidence angle and transmission angle. 
The simulated acoustic intensity at the PDMS/air interface is shown in Fig. 2. It can be seen that the intensity has relatively large values at the interface of PDMS/water, which means the energy is transmitted, while the intensity has relatively small values at the interface of PDMS/air, which means the energy is reflected. The acoustic energy will then focus at the designed focal point. The simulation result matches the practical experiment result.

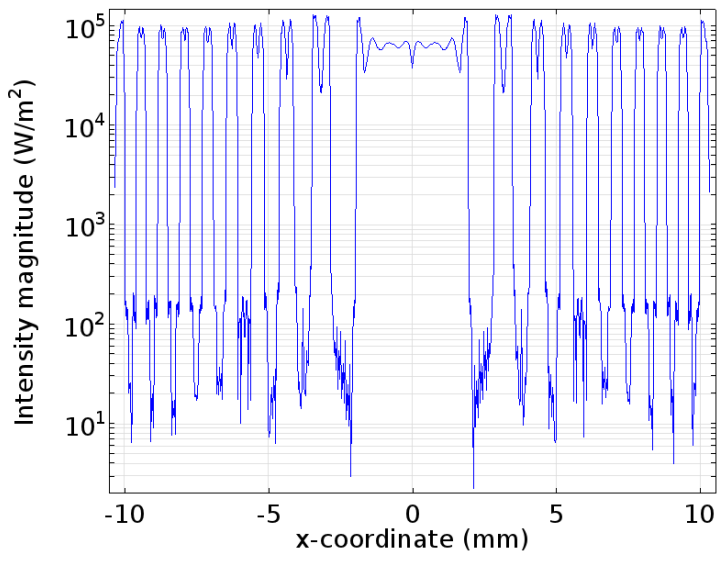

Fig.2 Simulated acoustic intensity at the PDMS/air interface.

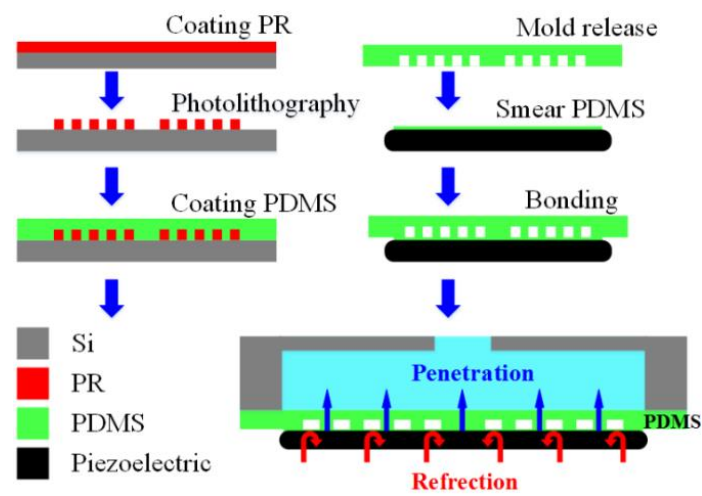

Fig.3 The fabrication process.

\section{FABRICATION}

For creating annular patterned membrane and fluidic channels, PDMS molding using SU-8 molds on silicon wafers is used. First, the molds were baked at $120{ }^{\circ} \mathrm{C}$ and coated with a mold releasing agent to enable the formed replicas to be released from the molds. Then, PDMS elastomer was mixed with its curing agent at a volume ratio of 10:1. The PDMS was then casted onto the prepared SU-8 mold with spinner and cured for 30 minutes at $120^{\circ} \mathrm{C}$. After cooling down, the PDMS membranes were stripped off from the molds carefully. Finally, the PDMS membrane is attached to the PZT transducer with uncured PDMS glue as an adhesive layer. The fabrication process is shown in Fig. 3. The fabricated PDMS membrane with the PZT transducer is shown in Fig.4. Fig.5 shows the measured surface profile of the SU-8 mold and the PDMS membrane.

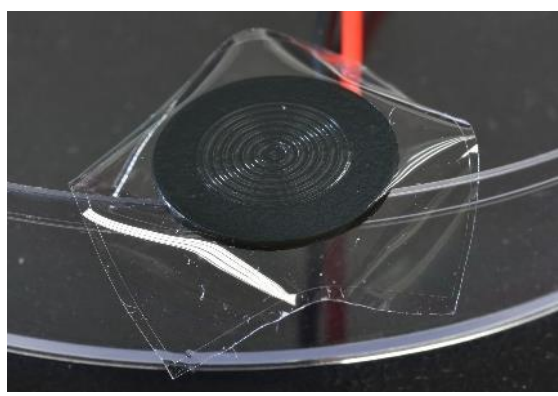

Fig.4 Fabricated patterned PDMS membrane with the PZT transducer.

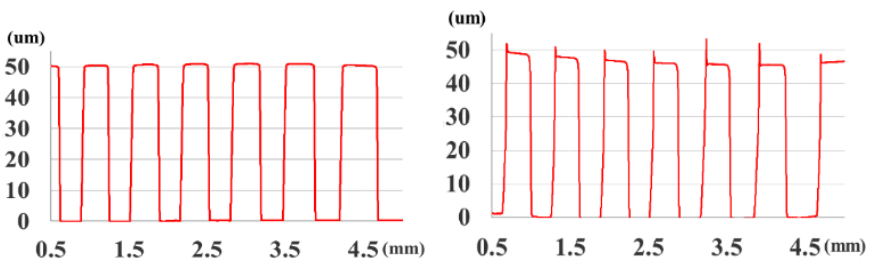

Fig.5 Measured surface profiles of the Fresnel lens membranes for the SU8 mold (left), and for the PDMS replica (right).

\section{INK STORAGE SYSTEM}

For downward ejection, a concentric circular chamber with a hole in the bottom for ink communication is designed for the ink storage, as shown in Fig.6. With communicating vessel principle and capillary theory, the liquid level in the ink storage system and ejector system are always kept at the same height. The liquid inside the ink storage chamber will be supplied to the ejector system until the liquid level is lower than the pressure balance chamber. The fabricated ink storage system is shown in Fig.7.

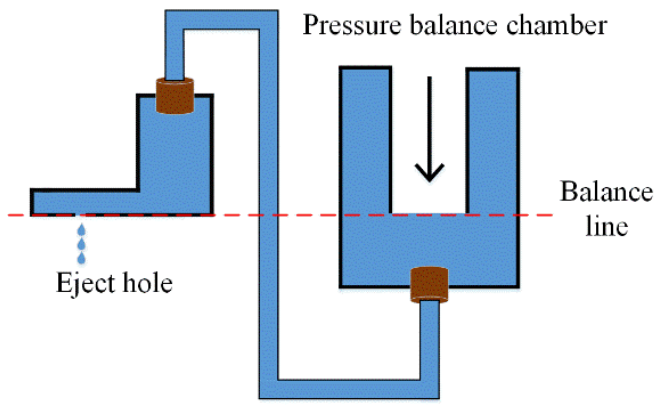

Fig.6 The schematic shows that liquid levels for the ink storage system and the ejector are kept the same.

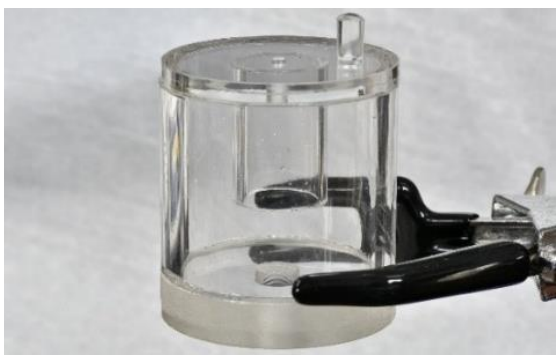

Fig.7 The acrylic ink storage system. 


\section{MEASUREMENT AND DISCUSSIONS}

In the measurement, an oscillating circuit is used to generate modulated driving signals of $2.4 \mathrm{MHz}$, as shown in Fig. 8. Every signal has a low-frequency region for $11 \mu$ s and a high frequency region for $17 \mu \mathrm{s}$, to satisfy the need of high speed rejection. A power amplifier increases the signal and a voltage of $70 \mathrm{~V}_{\mathrm{pp}}$ is applied to the PZT transducer.

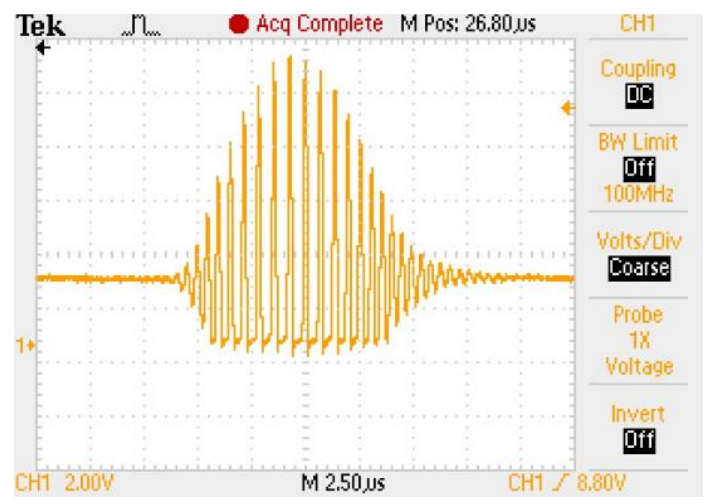

Fig.8 The signal generated by the oscillating circuit.

A high speed camera is used to record the dynamic images of the droplets for both upward and downward ejection. In the upward ejection experiment, the diameter of the ejected droplet reaches $920 \mu \mathrm{m}$ with a speed of $0.21 \mathrm{~m} / \mathrm{s}$, as shown in Fig.9. While in the downward ejection experiment, the diameter of the ejected droplet reaches $720 \mu \mathrm{m}$ with a speed of $0.29 \mathrm{~m} / \mathrm{s}$, as shown in Fig. 10. It could be seen that the upward ejection speed is lower than the downward ejection speed, and the upward droplet is larger than the downward droplet, due to the overcome of gravity. Fig.11 shows a single droplet jetting downward by the ejector.

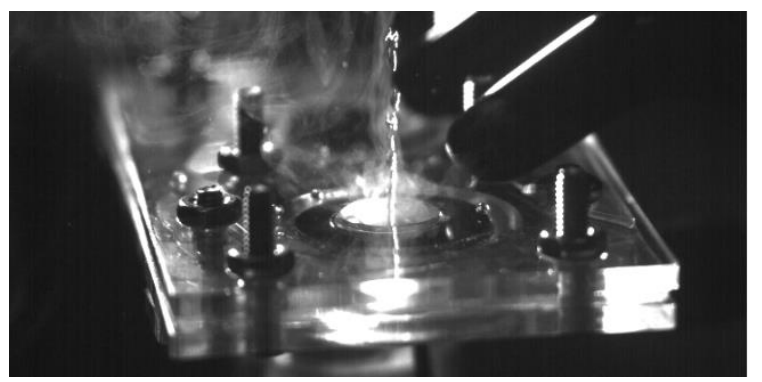

Fig.9 The upward ejecting droplet with a diameter of $920 \mu \mathrm{m}$ and a speed of $0.21 \mathrm{~m} / \mathrm{s}$

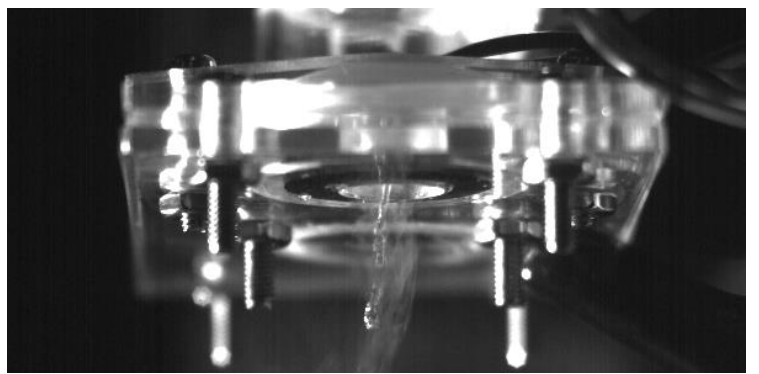

Fig.10 The downward ejecting droplet with a diameter of 720 $\mu \mathrm{m}$ and a speed of $0.29 \mathrm{~m} / \mathrm{s}$.

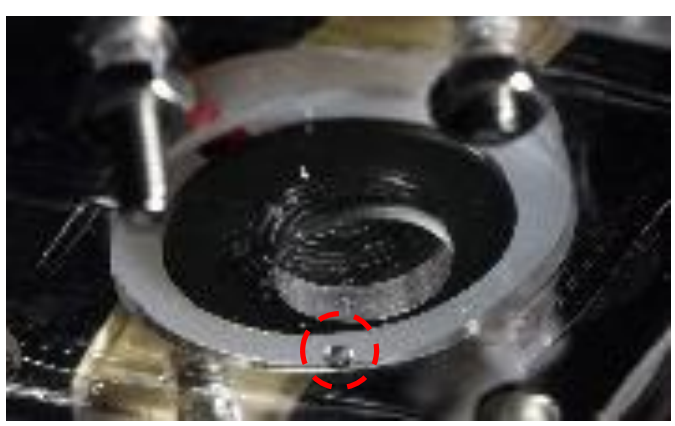

Fig.11 A photo of a single droplet jetting downward by the ejector.

\section{SUMMARY}

This paper reports a new acoustic-wave liquid ejector with a siphoning structure, which overcomes the limit of upward jetting direction and can automatically supply the ink without external electrical power. For long-term ejections, the focusing device is designed to be replaceable, for either annular patterned Fresnel lens membrane or piezoelectric transducer. In the experiment, the ejector can eject water droplet downward with a speed of $0.29 \mathrm{~m} / \mathrm{s}$ and size of $720 \mu \mathrm{m}$, and it could be used for better printing performance

\section{ACKNOWLEDGMENT}

The authors would like to thank National Center for Highperformance Computing, Taiwan, R.O.C. for providing software and facilities.

\section{REFERENCES}

[1] J. S. Aden, J. H. Bohórquez, D. M. Collins, and M. Douglas Crook, "The third-generation HP thermal inkjet printhead," Hewlett Packard Journal, vol. 45, pp. 41-41, 1994.

[2] A. Asai, T. Hara, and I. Endo, "One-dimensional model of bubble growth and liquid flow in bubble jet printers," Japanese Journal of Applied Physics, vol. 26, p. 1794, 1987.

[3] S. Hirata, Y. Ishii, H. Matoba, and T. Inui, "An ink-jet head using diaphragm microactuator," Proc. IEEE Micro Electro Mechanical Systems Workshop, San Diego, CA, Feb. 1996, pp. 418-423.

[4] S. Kamisuki, T. Hagata, C. Tezuka, Y. Nose, M. Fujii, and M. Atobe, "A low power, small, electrostatically-driven commercial inkjet head," Proc. IEEE Micro Electro Mechanical Systems Workshop, Heidelberg, Jan. 1998, pp. 63-68.

[5] G. Percin and B. T. Khuri-Yakub, "Piezoelectrically actuated flextensional micromachined ultrasound droplet ejectors," IEEE Transactions on Ultrasonics, Ferroelectrics, and Frequency Control, , vol. 49, pp. 756-766, 2002.

[6] D. Huang and E. S. Kim, "Micromachined acoustic-wave liquid ejector," Journal of Microelectromechanical Systems, , vol. 10, pp. 442-449, 2001.

[7] S. V. Murphy and A. Atala, "3D bioprinting of tissues and organs," Nature biotechnology, vol. 32, pp. 773-785, 2014.

[8] R. D. Farahani, K. Chizari, and D. Therriault, "Three-dimensional printing of freeform helical microstructures: a review," Nanoscale, vol. 6 , pp. 10470-10485, 2014.

[9] C.-Y. Lee, W. Pang, S. C. Hill, H. Yu, and E. S. Kim, "Airborne particle generation through acoustic ejection of particles-in-droplets," Aerosol Science and Technology, vol. 42, pp. 832-841, 2008. 